\title{
Evading death by vacuum
}

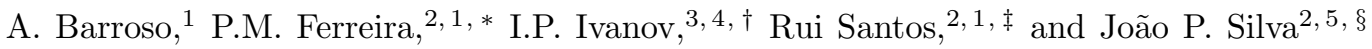 \\ ${ }^{1}$ Centro de Física Teórica e Computacional, Faculdade de Ciências, \\ Universidade de Lisboa, Av. Prof. Gama Pinto 2, 1649-003 Lisboa, Portugal \\ ${ }^{2}$ Instituto Superior de Engenharia de Lisboa, 1959-007 Lisboa, Portugal \\ ${ }^{3}$ IFPA, Université de Liége, Allée du 6 Août 17, bâtiment B5a, 4000 Liége, Belgium \\ ${ }^{4}$ Sobolev Institute of Mathematics, Koptyug avenue 4, 630090, Novosibirsk, Russia \\ ${ }^{5}$ Centro de Física Teórica de Partículas (CFTP), Instituto Superior Técnico, \\ Universidade Técnica de Lisboa, 1049-001 Lisboa, Portugal
}

(Dated: September 29, 2018)

\begin{abstract}
In the Standard Model, the Higgs potential allows only one minimum at tree-level. But the open possibility that there might be two scalar doublets enriches the vacuum structure, allowing for the risk that we might now be in a metastable state, which we dub the panic vacuum. Current experiments at the LHC are probing the Higgs particle predicted as a result of the spontaneous symmetry breaking. Remarkably, in the two Higgs model with a softly broken $U(1)$ symmetry, the LHC experiments already preclude panic vacuum solutions.
\end{abstract}

PACS numbers: 12.60.Fr, 14.80.Ec, 11.30.Qc, 11.30.Ly

The Standard Model (SM) of particle physics is a remarkably successful theory. Over the past forty years, the SM has passed numerous tests, predicting, and agreeing with, experimental observables with great accuracy. A crucial element of the Model is the mechanism of spontaneous symmetry breaking (SSB) through which the elementary particles acquire their masses, and which necessitated the introduction of a new particle, known as the Higgs scalar 2. But this mechanism has only recently come under experimental scrutiny, with the discovery by the LHC collaborations ATLAS and CMS of a particle with properties similar to those expected for the SM Higgs 3. The particle is produced by colliding two protons $(p p)$ and detected, at present, mostly through its decay into two photons $(\gamma \gamma)$ and its decay into two $Z$ bosons $(Z Z)$. It turns out that the specific production mechanism can sometimes be tagged and that it affects the detectability. In the case of $\gamma \gamma$, results are known for all production mechanisms combined, and also for production of the Higgs through the fusion of two $Z$ or of two $W$ bosons, known collectively as vector boson fusion (vbf).

The field content of the SM has been determined by experiment. Indeed, $\mathrm{CP}$ violation aside, the theory would have been viable if there were only one charged lepton, one neutrino, one up-quark, and one down-quark. But three such families have been found, with increasing masses. In the same fashion, there may be more than one scalar doublet, and only experiment will tell. The two Higgs doublet model was proposed by Lee [5, 6] in 1973 as a means of trying to explain the matter-antimatter

\footnotetext{
*E-mail: ferreira@cii.fc.ul.pt

${ }^{\dagger}$ E-mail: igor.ivanov@ulg.ac.be

${ }^{\ddagger}$ E-mail: rsantos@cii.fc.ul.pt

$\S$ E-mail: jpsilva@cftp.ist.utl.pt
}

asymmetry in the universe. It is a very compelling generalization of the SM, with a richer scalar spectrum: there would be three neutral scalars ( $h$ and $H$, respectively the lightest and heaviest CP-even scalars; and $A$, the pseudoscalar); and a pair of conjugate charged scalars $\left(H^{ \pm}\right)$. But having more than one scalar doublet also enriches the vacuum structure. In the SM with one scalar doublet, the potential admits only one minimum at treelevel, up to gauge transformations. Nevertheless, quantum corrections may induce vacuum metastability - in fact, the running of the scalar quartic coupling can be driven to negative values at very high energy scales, due to the largeness of the top quark Yukawa. This would correspond to the existence of a deeper minimum of the potential, and "ours" would be a false vacuum. Recent calculations have shown that, with a Higgs mass around $125 \mathrm{GeV}$, the scalar self-coupling would become negative well below the Planck mass - depending on the uncertainties on the top quark pole mass and on the strong coupling constant, this would occur between $10^{10}$ and $10^{14} \mathrm{GeV}$ [4.

In the 2HDM there is the possibility of metastable vacua even at tree-level. In fact, the vacuum structure of the $2 \mathrm{HDM}$ is much richer than the SM's: for instance, charge breaking $(\mathrm{CB})$ and $\mathrm{CP}$ breaking minima can occur in the 2HDM, but not in the SM. It has been proven 8,9 that no minima of different natura can coexist in the 2HDM: if a charge and $\mathrm{CP}$ preserving minimum exists, then a $\mathrm{CB}$ or CP stationary point is necessarily a saddle point, and lies above it. And if a $\mathrm{CP}$ (or $\mathrm{CB}$ ) minimum occurs, all other types of stationary points have to be saddle points. Nonetheless, a different possibility occurs: the $2 \mathrm{HDM}$ can have two minima which preserve $\mathrm{CP}$, but break the standard electroweak gauge symmetries. And those two minima can be non-degenerate, corresponding to a completely different mass spectrum for all elementary particles in each of them. And again, we stress that this situation arises at tree-level. Radiative corrections 
will undoubtedly have relevant contributions to make, but the possibility of metastability in the $2 \mathrm{HDM}$ is potentially more serious than in the SM.

In fact, vacuum metastability raises a troubling possibility. If we were at present in the metastable vacuum, then the scalar field could in time decay into the real vacuum. Since in the deeper vacuum the scalar fields' vacuum expectation values have in general very different values, tunneling to the true vacuum of the model would alter all particle masses, with dramatic consequences for the entire universe. We call this situation a "panic vacuum". In this paper, we will consider one of the simplest versions of the $2 \mathrm{HDM}$ where this situation might occur the Peccei-Quinn model. We will present the conditions that the parameters of the theory need to obey in order to avoid all tree-level metastability. And we will show that, remarkably, the current experimental results from the LHC can already be used to probe this question. In fact, they can already exclude, at the $2 \sigma$ level, any possibility of panic vacua. We will also present an estimate of the lifetime of the false vacua, and show that most of them would have a tunneling time inferior to the age of the universe. Nonetheless, the LHC is already telling us that the Peccei-Quinn model, if it describes particle physics, has a vacuum which is completely stable at treelevel.

\section{FALSE VACUA IN THE PECCEI-QUINN MODEL}

In this paper we will study one of the simplest possible versions of the $2 \mathrm{HDM}$, the Peccei-Quinn model [7, 6]. This model has a global $U(1)$ symmetry, such that the scalar fields transform as $\left(\Phi_{1}, \Phi_{2}\right) \rightarrow\left(\Phi_{1}, e^{i \xi} \Phi_{2}\right)$. Extending this symmetry to the Yukawa sector, it is possible to make sure that the model possesses no tree-level favour changing neutral currents (which would be present otherwise). It also considerably simplifies the scalar potential, which is given by

$$
\begin{aligned}
V_{H} & =m_{11}^{2} \Phi_{1}^{\dagger} \Phi_{1}+m_{22}^{2} \Phi_{2}^{\dagger} \Phi_{2}-m_{12}^{2}\left[\Phi_{1}^{\dagger} \Phi_{2}+\Phi_{2}^{\dagger} \Phi_{1}\right] \\
& +\frac{1}{2} \lambda_{1}\left(\Phi_{1}^{\dagger} \Phi_{1}\right)^{2}+\frac{1}{2} \lambda_{2}\left(\Phi_{2}^{\dagger} \Phi_{2}\right)^{2} \\
& +\lambda_{3}\left(\Phi_{1}^{\dagger} \Phi_{1}\right)\left(\Phi_{2}^{\dagger} \Phi_{2}\right)+\lambda_{4}\left(\Phi_{1}^{\dagger} \Phi_{2}\right)\left(\Phi_{2}^{\dagger} \Phi_{1}\right),
\end{aligned}
$$

where all parameters are real because $\mathrm{CP}$ invariance is imposed. Notice that we have included a soft breaking term - the $m_{12}^{2}$ term - to prevent the appearance of a massless pseudoscalar (an axion) when both doublets acquire a vacuum expectation value (vev).

In this model, spontaneous $\mathrm{CP}$ breaking cannot occur. And since the electromagnetic symmetry is unbroken, this means that, after SSB, only the neutral components of the scalars will develop vevs. That is, $\left\langle\Phi_{1}^{0}\right\rangle=v_{1} / \sqrt{2}$ and $\left\langle\Phi_{2}^{0}\right\rangle=v_{2} / \sqrt{2}$, which can be traded for $v=\sqrt{v_{1}^{2}+v_{2}^{2}}$ and $\tan \beta=v_{2} / v_{1}$. Without loss of generality, we can take $v_{1}$ and $v_{2}$ positive and $0 \leq \beta \leq$ $\pi / 2$. The vacuum that our Universe is currently in has $v=2 m_{W} / g=246 \mathrm{GeV}$ (related to the $W$ mass and the weak coupling $g$ ). Now, it has been shown [10, 11] that the $2 \mathrm{HDM}$ can in fact have at most two minima of this kind - the first with the vevs $v_{1}$ and $v_{2}$ defined above, and the second with vevs $v_{1}^{\prime}$ and $v_{2}^{\prime}$, such that

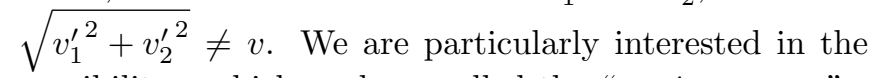
possibility - which we have called the "panic vacuum" that the vacuum we are living in is not the deepest one. The second set of vevs would thus correspond to a lower potential,

$$
V_{H}\left(v_{1}^{\prime}, v_{2}^{\prime}\right)<V_{H}\left(v_{1}, v_{2}\right) .
$$

The vevs can be obtained by minimizing $V_{H}$, leading to two coupled cubic equations. Given $m_{11}^{2} \ldots \lambda_{4}$, these can be solved numerically. Fortunately, many interesting features of the vacuum can be determined without solving those equations explicitly. In fact, in Ref. [10] a general study of the conditions under which these two minima arise was undertaken, and conditions for their existence, and relative depth, were established. These are re-derived and expanded in a parallel paper [12]. Applying the results of 10 to the specific case of the softly broken Peccei-Quinn model, the two minima can occur only if

$$
\begin{aligned}
m_{11}^{2}+k^{2} m_{22}^{2} & <0 \\
\sqrt[3]{x^{2}}+\sqrt[3]{y^{2}} & \leq 1
\end{aligned}
$$

where

$$
\begin{aligned}
& x=\frac{4 k m_{12}^{2}}{m_{11}^{2}+k^{2} m_{22}^{2}} \frac{\sqrt{\lambda_{1} \lambda_{2}}}{\lambda_{34}-\sqrt{\lambda_{1} \lambda_{2}}}, \\
& y=\frac{m_{11}^{2}-k^{2} m_{22}^{2}}{m_{11}^{2}+k^{2} m_{22}^{2}} \frac{\sqrt{\lambda_{1} \lambda_{2}}+\lambda_{34}}{\sqrt{\lambda_{1} \lambda_{2}}-\lambda_{34}}
\end{aligned}
$$

with

$$
\lambda_{34}=\lambda_{3}+\lambda_{4} \quad \text { and } \quad k=\sqrt[4]{\frac{\lambda_{1}}{\lambda_{2}}}
$$

The limiting curve $\sqrt[3]{x^{2}}+\sqrt[3]{y^{2}}=1$ is known as an astroid. If either of conditions (3) or (4) are not verified, then the potential has a single minimum (which is of course the global minimum of the potential).

However, in the region where two minima can coexist, one must investigate whether the minimum with vevs $v_{1}$, $v_{2}$ is, or isn't, the global minimum of the model. It turns out [10, 12] that answering that question ends up being very simple. All one has to do is to define the following quantity, which we call a discriminant,

$$
D=\left(m_{11}^{2}-k^{2} m_{22}^{2}\right)(\tan \beta-k) .
$$

The discriminant is, of course, computed in our vacuum. Then, the following theorem holds: 
Our vacuum is the global minimum of the theory if and only if $D>0$.

If, on the other hand, we find that $D<0$, then Eq. (2) holds and we live in a metastable state, and our current $\left(v_{1}, v_{2}\right)$ solution is the "panic vacuum".

Conversely, if there is only one minimum, or even if there are two minima - Eqs. (3) and (4) hold - but $D>0$ in the region where it is defined, then $\left(v_{1}, v_{2}\right)$ corresponds to the global minimum of the theory, and our vacuum is stable. This means that, to ascertain the nature of the Peccei-Quinn model vacuum, all one truly needs is to verify the value of the discriminant $D$.

Problems related to ours are sometimes tackled by following the evolution of the various minima as the temperature $(T)$ of the Universe is decreased, from its inception to the present day. This involves estimates of the finite temperature contributions to the effective potential, and hinges on a variety of assumptions. Some recent work within the $2 \mathrm{HDM}$ can be found in Ref. 13. It is also true that, even at $T=0$, the nature of the vacuum changes as we increase the energy scale above $10 \mathrm{TeV}$. As we mentioned earlier, this occurs even in the SM, due to loop corrections to $V_{H}$, and might ultimately point to a more complete theory at high energies, encompassing the SM [4. This is not our concern here, since we are worried about a problem that affects us right now, at $T=0$ and small energies. One might expect that radiative corrections will be important in the $2 \mathrm{HDM}$, as they are in the SM. In fact, they might even raise the possibility of further deeper minima existing. But the issue in the $2 \mathrm{HDM}$ is more immediate, since both minima exist at tree level and $T=0$.

\section{PANIC VACUA AND LHC RESULTS}

We will now show that measurements in particle physics experiments can be used to reconstruct the shape of the present day $(T=0)$ tree-level scalar potential, and, thus, ascertain as to the putative presence of a lower lying minimum. Even more striking, although there are not, as of yet, enough measurements to determine all the seven $m_{11}^{2} \ldots \lambda_{4}$ parameters of $V_{H}$ - or even any indication of the existence of more than one scalar - the experiments at the LHC are already close to ruling out the panic vacuum situation of Eq. (2). In fact, they strongly disfavour the possibility of a panic vacuum at the $2 \sigma$ level.

If the soflty broken Peccei-Quinn model describes Nature, and the three neutral scalars and conjugate pair of charged scalars are detected, then parameters of the scalar potential can be reproduced from the following re- lations,

$$
\begin{aligned}
m_{12}^{2} & =m_{A}^{2} s_{\beta} c_{\beta}, \\
\lambda_{1} & =\frac{-s_{\beta}^{2} m_{A}^{2}+c_{\alpha}^{2} m_{H}^{2}+s_{\alpha}^{2} m_{h}^{2}}{v^{2} c_{\beta}^{2}}, \\
\lambda_{2} & =\frac{-c_{\beta}^{2} m_{A}^{2}+s_{\alpha}^{2} m_{H}^{2}+c_{\alpha}^{2} m_{h}^{2}}{v^{2} s_{\beta}^{2}}, \\
\lambda_{3} & =\frac{2 m_{H^{ \pm}}^{2}-m_{A}^{2}}{v^{2}}+\frac{s_{2 \alpha}\left(m_{H}^{2}-m_{h}^{2}\right)}{v^{2} s_{2 \beta}}, \\
\lambda_{4} & =\frac{2\left(m_{A}^{2}-m_{H^{ \pm}}^{2}\right)}{v^{2}},
\end{aligned}
$$

where $c_{\theta}=\cos \theta$ and $s_{\theta}=\sin \theta$, for any needed angle $\theta$, and the $m$ 's are the masses of the various scalars. The $m_{11}^{2}$ and $m_{22}^{2}$ parameters are obtained by solving the stationarity conditions $\partial V_{H} /\left(\partial v_{1}\right)=0$ and $\partial V_{H} /\left(\partial v_{2}\right)=0$. The angle $\alpha$ parametrizes the mixing

$$
\begin{aligned}
h & =\operatorname{Re}\left(\Phi_{1}^{0}\right) s_{\alpha}-\operatorname{Re}\left(\Phi_{2}^{0}\right) c_{\alpha}, \\
H & =-\operatorname{Re}\left(\Phi_{1}^{0}\right) c_{\alpha}-\operatorname{Re}\left(\Phi_{2}^{0}\right) s_{\alpha},
\end{aligned}
$$

and can be taken between $-\pi / 2$ and $\pi / 2$. Eventually, the angles $\alpha$ and $\beta$ will be determined by probing the couplings of the scalar particles to the fermions. Usually, two types of couplings to fermions are considered in theories with the $U(1)$ symmetry. We thus consider two different models, with the same scalar potential but different scalar-fermion interactions. In model Type-I, the fermions transform under the Peccei-Quinn symmetry in such a manner that all fermions couple only to the $\Phi_{2}$ doublet. But in model Type-II, the fermion's transformation laws are chosen such that up-quarks couple to $\Phi_{2}$, while down-quarks and charged leptons couple to $\Phi_{1}$. Both of these types of models have interesting phenomenologies, and are constrained in different manners by existing data, mostly coming from B-physics experiments [14.

It could happen that the panic vacua solutions could only occur for uninteresting regions of parameter space - for instance, if they could only occur for, say a Higgs mass of $50 \mathrm{GeV}$, we could dismiss metastable vacua in the Peccei-Quinn model as being only a theoretical curiosity. In order to probe the interest of the panic vacua solutions we have therefore performed a vast scan of the parameter space of the model and verified where those solutions arose. We have randomly generated 180000 points in the parameter space - since $v$ and $m_{h}$ are fixed, each "point" corresponds to a combination of five different parameters. Ours is therefore a 5 -dimensional parameter space, and all plots we present are to be understood as "slices" of that higher-dimensional space. In our scan, we set $v=246 \mathrm{GeV}$ and $m_{h}=125 \mathrm{GeV}$, and varied the remaining parameters in the ranges $m_{H} \in[125,900] \mathrm{GeV}$, $m_{A} \in[10,900] \mathrm{GeV}, m_{H^{ \pm}} \in[90,900] \mathrm{GeV}$, and $-\pi / 2 \leq$ $\alpha \leq \pi / 2$. In addition, due to experimental bounds coming from $B$-physics [14, we have kept $\tan \beta>1$. The corresponding couplings in Eqs. (8) have been forced to 
comply with a scalar potential bounded from below [15], to satisfy tree-level unitarity [16, 17], and to be consistent with constraints from the electroweak precision observables $S, T$ and $U$ [18, 19].

How often can both minima exist in this model? To verify this, we have computed the $x$ and $y$ variables of Eqs. (5) for each combination of parameters in the potential and plot the value of Eq. (4) in Fig. 1. There we show the generated points in the $(x, y)$ plane and in

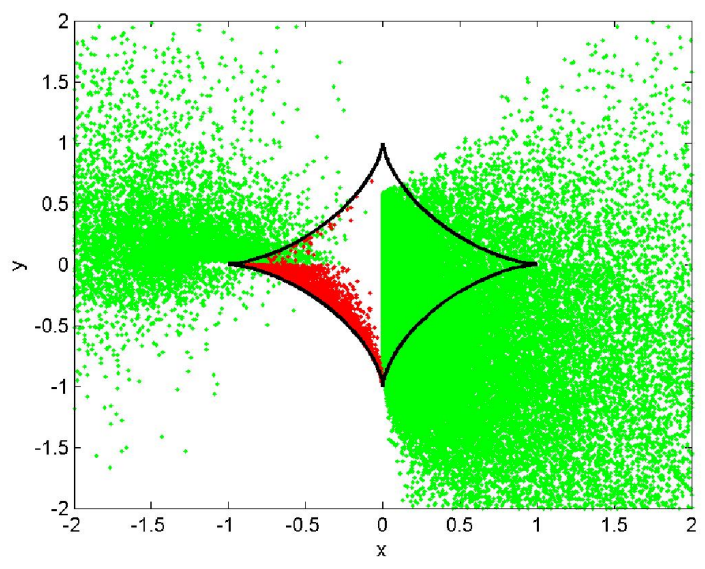

FIG. 1: Placement in the $(x, y)$ plane of the points generated. Points inside the astroid (solid lines) can have two minima; those in red/dark-gray correspond to the panic vacuum.

solid black lines the astroid which delimits the region where dual minima may exist. Points inside (outside) the astroid satisfy (do not satisfy) Eq. (4) and correspond to potentials which do (do not) have two minima. Green/light-gray points inside the astroid have $D>0$ and exhibit two minima, with our current vacuum being the global minimum. This is a safe situation, no tunneling from our vacuum to another can occur (at tree level). Red/dark-gray points inside the astroid have $D<0$, exhibit two minima, but correspond to the panic situation where our current vacuum lies above the other one. We have checked by explicit computation that the red/darkgray points satisfy Eq. (2), while the green/light-gray points inside the astroid do not. As we see from Fig. 1 , the occurrence of two minima is not a rare event in the 2HDM - a "blind" scan of parameters encounters many such minima, and among them the panic vacua aren't rare either.

In order to study the panic vacuum in detail, we have generated two new sets of points where Eqs. (3)-(4) hold, and $D<0$. The data sample for model Type-I has over 100000 points. In the Type-II model we have a further constraint - the charged scalar mass has to be such that $m_{H^{ \pm}}>340 \mathrm{GeV}$. This arises from $b \rightarrow s \gamma$ measurements, and is almost independent of $\tan \beta[14]$. For model Type-II we have thus generated 58000 points obeying this further constraint, as well as all the other mentioned pre- viously.

The generated points are used to calculate $R_{f}$, defined as the number of events predicted in the model for the process $p p \rightarrow h \rightarrow f$, divided by the prediction obtained in the SM for the same final state $f$. In other words,

$$
R_{f}=\frac{\sigma^{2 H D M}(p p \rightarrow h) B R^{2 H D M}(h \rightarrow f)}{\sigma^{S M}(p p \rightarrow h) B R^{S M}(h \rightarrow f)},
$$

with production cross sections $\sigma$ and the branching ratios (BR) of the Higgs boson considered for both models. We have considered all Higgs production mechanisms which are at work at LHC: gluon-gluon (gg) fusion; vector boson fusion (vbf); associated production of a Higgs and a vector boson or a $t \bar{t}$ pair; and $b \bar{b}$ fusion. The two LHC observables which give more precise results for Higgs physics at the moment are the $R$ ratios to two photons or two $Z$ bosons. Their experimental bounds at the $1 \sigma$ level are [21] $R_{Z Z}=0.93 \pm 0.28, R_{\gamma \gamma}=1.66 \pm 0.33$, summing over all production mechanisms.

The results we obtain for Type-II are shown in Figs. 2 and 3 Fig. 3 also includes the $1 \sigma$ (solid line) and $2 \sigma$

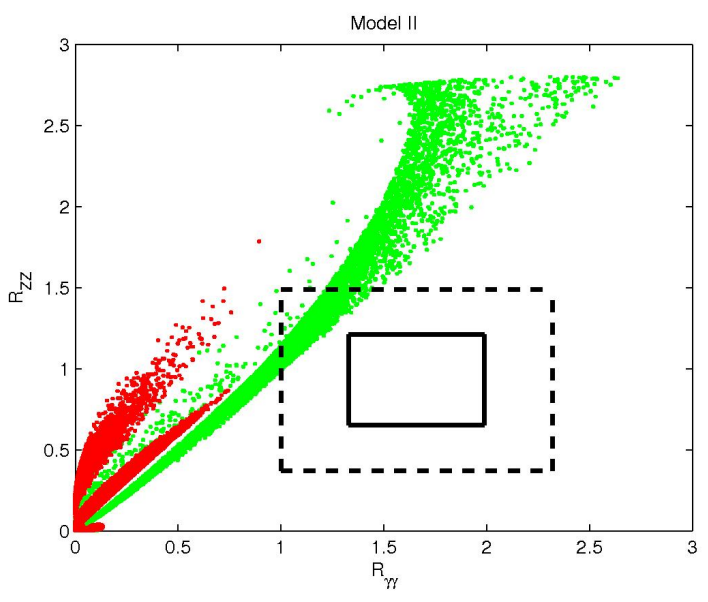

FIG. 2: Predictions from Type-II in the $\left(R_{\gamma \gamma}, R_{Z Z}\right)$ plane for panic vacuum points (in red/dark-gray) and for non-panic points (in green/light-gray). Also shown are the $1 \sigma$ (solid line) and $2 \sigma$ (dashed line) experimental bounds.

(dashed line) bounds coming from the ellipse in Fig. 12 of Ref. [20]. While model Type-II points (green/light-gray) for which our current vacuum coincides with the global minimum are consistent with experiment, we find that model Type-II panic points (red/dark-gray) are excluded at least at the $2 \sigma$ level by both $Z Z$ and by $\left(R_{\gamma \gamma}, R_{\gamma \gamma}^{\text {vbf }}\right)$. Since our parameter space is 5-dimensional, it is very hard to find a simple-to-grasp explanation as to why the panic points, which obey $D<0$ as well as Eqs. (3) and (4), should concentrate as they do, for lower values of $R_{\gamma \gamma}$. But the numerical scan shows they do, and as such are strongly disfavoured by LHC data.

The results we obtain for model Type-I are shown in the $\left(R_{\gamma \gamma}, R_{Z Z}\right)$ plane of Fig. 4. We find that $R_{\gamma \gamma} \lesssim 0.88$, 


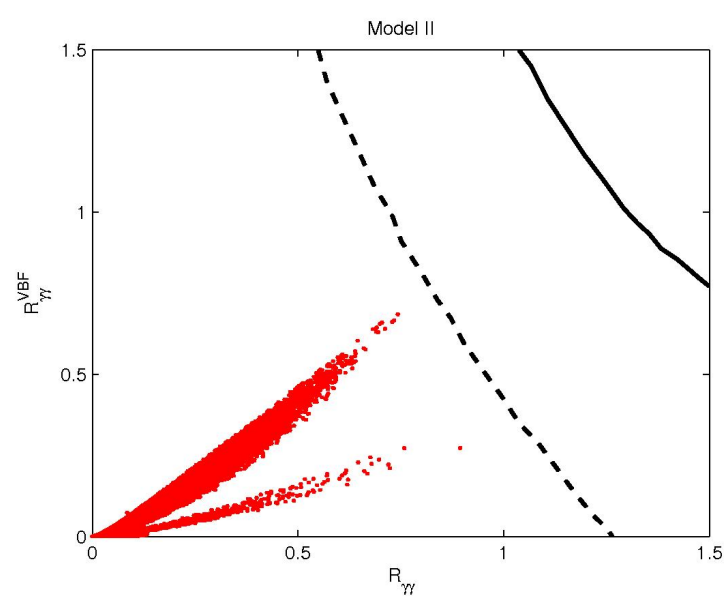

FIG. 3: Prediction for panic vacuum points in Type-II in the $\left(R_{\gamma \gamma}, R_{\gamma \gamma}^{\mathrm{vbf}}\right)$ plane. They lie outside the $1 \sigma$ (solid line) and $2 \sigma$ (dashed line) ellipse bounds in Fig. 12 of Ref. 20].

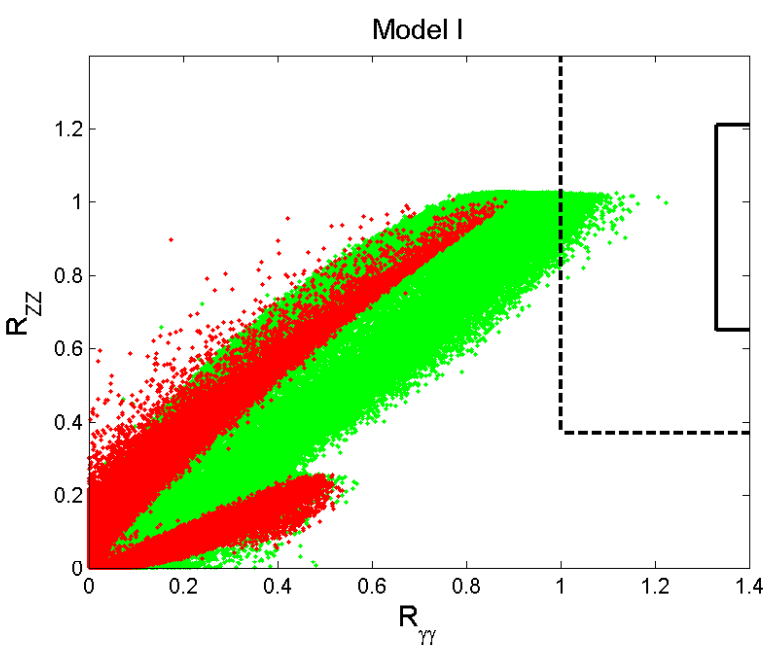

FIG. 4: Predictions from Type-I in the $\left(R_{\gamma \gamma}, R_{Z Z}\right)$ plane for panic vacuum points (in red/dark-gray) and for non-panic points (in green/light-gray). Also shown are the $1 \sigma$ (solid line) and $2 \sigma$ (dashed line) experimental bounds.

$R_{Z Z} \lesssim 1$, and the panic points lie outside the $2 \sigma R_{\gamma \gamma}$ experimental band. In contrast, the Type-I panic points we have generated in the $\left(R_{\gamma \gamma}, R_{\gamma \gamma}^{\mathrm{vbf}}\right)$ plane lie outside the $1 \sigma$ (elliptical) band, but inside the $2 \sigma$ band. We could have $R_{\gamma \gamma}^{\mathrm{vbf}}>1$, but only for values of $R_{\gamma \gamma}<0.6$. Thus, we cannot reach the SM values, $R_{\gamma \gamma}=R_{Z Z}=R_{\gamma \gamma}^{\mathrm{vbf}}=1$, and the measurements of $R_{\gamma \gamma}$ are barely consistent with our results for Type-I.

\section{FALSE VACUUM LIFETIME ESTIMATES}

Thus far, we have taken the view that, regardless of how one estimates the Universe's evolution, the possi- bility that we are now (or not) in a metastable vacuum can be ascertained by measurements in particle physics experiments. But it is interesting to have an estimate of what cosmology has to say about the likelihood of a panic vacuum in our model. In fact, one usually takes the point of view that, if the tunneling time between the false vacuum and the true one is larger than the current age of universe, then the parameters of the potential which produce said false vacuum are perfectly acceptable. In fact, such a situation would be indistinguishable from our vacuum being the model's global minimum. In a theory with a single scalar, the lifetime of the false vacuum is estimated calculating the bounce trajectory between both minima 22. That trajectory passes through the maximum between both minima. The estimate of the vacuum lifetime is however very difficult in the $2 \mathrm{HDM}$, given that there are many field directions present. In fact, even removing the would-be Goldstone bosons, the scalar potential is still a five-variable function. Thus, the path from one minimum to the other can be much "shorter" than what one would expect. In fact, it doesn't even need to pass by the potential's maximum, it can pass by a saddle point, or in fact avoid any stationary point whatsoever [23]. We will follow the standard procedure and take the thin wall approximation of Refs. 222 24. We therefore estimate the decay width of the false vacuum per unit volume as the exponential of

$$
-B=-\frac{2^{11} \pi^{2}}{3 \lambda}\left(\frac{\delta}{\epsilon}\right)^{3} .
$$

In this formula $\delta$ is the height of the barrier at the saddle point between the two minima, relative to the highest minimum - in this way we are trying be be conservative and taking the most unfavourable estimate, since this saddle point is actually lower than the potential's maximum, thus quickening the tunneling time. As for $\epsilon$, it is the difference of the values of the potential calculated in the two minima. To further make sure our estimate is the most conservative one, we take $\lambda$ as the largest of the quartic couplings in the scalar potential, $\lambda=\max \left(\left|\lambda_{k}\right|\right)$ $(k=1 \ldots 5)$. Having $\delta / \epsilon$ larger than order one is already sufficient to stabilize the Universe; for example, Ref. 25] quotes $B>400$. Thus, large values of $\delta / \epsilon$ correspond to a tunneling time to the deeper vacuum larger than the age of the Universe.

In order to perform this lifetime estimate, we generated a separate sample of points, consisting only of panic vacua points, and for which we had all information concerning both vacua: namely, both sets of vevs $\left\{v_{1}, v_{2}\right\}$ and $\left\{v_{1}^{\prime}, v_{2}^{\prime}\right\}$. This is simple to achieve in a numerical way, although it would be impossible to present analytical expressions for both sets of vevs. It is further possible, employing the Lagrange multiplier formalism used in [10], to discover the remaining stationary points of the potential, and thus find the closest saddle point to the panic vacuum. In this manner we can obtain the values of both $\delta$ and $\epsilon$.

With that calculation, we have been able to show that, 
out of all the points shown in Fig. 1, only about $5 \%$ have estimated lifetimes which are larger than the current age of the universe. They are located close to the axis of the astroid. In contrast, points with small values of $\delta / \epsilon$ correspond to a tunneling time to the deeper vacuum smaller than the age of the Universe. These points must be excluded from the parameter space on cosmological grounds, because they would correspond to a situation in which the Universe would already have decayed away into the global minimum. The lifetime estimate we performed thus shows that the vast majority of the panic vacua one finds are indeed unacceptable choices of parameters, since they predict a vacuum which would have decayed long ago. However, we must stress our point: although these cosmological estimates preclude a good portion (but not all) of the panic vaccua, they hinge on the thin wall approximation that breaks down precisely when $\delta / \epsilon$ is very small. One could argue that a more accurate (and much more complicated) calculation of the lifetime could significantly shift its numerical values. As such, we cannot, based on this simple calculation, be certain which part of the astroid can be disregarded on cosmological grounds, and which is cosmologically viable. In contrast, current LHC bounds allow us to already exclude all panic vacuum points, regardless of any lifetime estimate. The beautiful thing in this model is that the lifetime estimate ends up being unnecessary, since LHC data already tells us that the Peccei-Quinn vacuum is a global one.

\section{CONCLUSIONS}

We have studied the vacuum structure of a $2 \mathrm{HDM}$ with a softly broken $U(1)$ symmetry, looking out for situations where our current vacuum has a larger energy density than the true global minimum of the scalar potential. We stress that the exclusion of panic vacuua should be addressed in models with extended Higgs sectors, where it should be implemented as an extra constraint on the theory's parameter space. In fact, the conditions for panic vacua we present in this work are a special case of those studied in ref. 12]. The discriminant we presented in this paper, Eq. $(7)$, is the only quantity we need to compute to ascertain whether a vacuum of the softly broken Peccei-Quin model is, or isn't the global minimum of the potential.

What we have found is that, in this model, the current LHC results allow us to conclude, at the $2 \sigma$ level, that it is impossible that we are currently living in the higher minimum - even without evidence of any extra scalars, the LHC already provides us information about the nature of the $2 \mathrm{HDM}$ vacuum, which is quite remarkable. For many of the parameter points we found in which panic vacua occurred - that is, where our vacuum would be the highest minimum of the potential - a standard estimation of the tunneling time to the lower minimum tells us that we could no longer be there. That is, most of the panic vacua we found, characterized by $D<0$, are (according to standard vacuum lifetime estimates) phenomenologically unacceptable, as they predict a vacuum which would have decayed long ago. Hence, such parameters could, on cosmological grounds, be excluded from the model's parameter space. But an interesting aspect of our analysis is that we need not even worry about vacuum lifetimes - the LHC data already enables us to exclude panic vacuum solutions.

Our conclusion is that, within the softly broken PecceiQuinn model, the recent LHC experiments are crucial in excluding the panic vacuum in model Type-II and strongly disfavoring it in model Type-I.

\section{Acknowledgments}

The works of A.B., P.M.F. and R.S. are supported in part by the Portuguese Fundação para a Ciência e a Tecnologia (FCT) under contract PTDC/FIS/117951/2010, by FP7 Reintegration Grant, number PERG08-GA2010-277025, and by PEst-OE/FIS/UI0618/2011. The work of J.P.S. is funded by FCT through the projects CERN/FP/109305/2009 and U777-Plurianual, and by the EU RTN project Marie Curie: PITN-GA-2009237920 .
[1] S. L. Glashow, Nucl. Phys. B 22, 579 (1961). S. Weinberg, Phys. Rev. Lett. 19, 1264 (1967). A. Salam, Elementary Particles and Fields: Relativistic Groups and Analiticity (Nobel Symposium No. 8), edited by N. Svartholm (Almquist and Wiksell, Stockholm, 1968), p. 367.

[2] P. W. Higgs, Phys. Lett. 12, 132 (1964). F. Englert and R. Brout, Phys. Rev. Lett. 13, 321 (1964). P. W. Higgs, Phys. Rev. Lett. 13, 508 (1964). G. S. Guralnik, C. R. Hagen and T. W. B. Kibble, Phys. Rev. Lett. 13, 585 (1964).

[3] For recent results, see G. Aad et al. [ATLAS Collaboration], Phys. Lett. B 716, 1 (2012) arXiv:1207.7214 [hep- ex]]. S. Chatrchyan et al. [CMS Collaboration], Phys. Lett. B 716, 30 (2012) arXiv:1207.7235[hep-ex]].

[4] G. Degrassi, S. Di Vita, J. Elias-Miro, J. R. Espinosa, G. F. Giudice, G. Isidori and A. Strumia, JHEP 1208, 098 (2012) arXiv:1205.6497[hep-ph]].

[5] T. D. Lee, Phys. Rev. D 8 (1973) 1226.

[6] For a recent review on the 2HDM, see, for example, G.C. Branco, P.M. Ferreira, L. Lavoura, M.N. Rebelo, M. Sher and J.P. Silva, Phys. Rept. 516, 1 (2012) arXiv:1106.0034 [hep-ph]].

[7] R. D. Peccei and H. R. Quinn, Phys. Rev. Lett. 38 (1977) 1440.

[8] P. M. Ferreira, R. Santos and A. Barroso, Phys. Lett. 
B 603 (2004) 219 [Erratum-ibid. B 629 (2005) 114] arXiv:hep-ph/0406231.

[9] A. Barroso, P. M. Ferreira and R. Santos, Phys. Lett. B 632 (2006) 684 arXiv:hep-ph/0507224.

[10] I. P. Ivanov, Phys. Rev. D 75, 035001 (2007) [Erratumibid. D 76, 039902 (2007)] hep-ph/0609018; ibid, 77, 015017 (2008).

[11] A. Barroso, P. M. Ferreira and R. Santos, Phys. Lett. B 652, 181 (2007) hep-ph/0702098 [HEP-PH]].

[12] A. Barroso, P. M. Ferreira I. P. Ivanov and R. Santos, to be published.

[13] N. Turok and J. Zadrozny, Nucl. Phys. B 369, 729 (1992). I. P. Ivanov, Acta Phys. Polon. B 40, 2789 (2009) arXiv:0812.4984 [hep-ph]]. I. F. Ginzburg, I. P. Ivanov and K. A. Kanishev, Phys. Rev. D 81, 085031 (2010) arXiv:0911.2383 [hep-ph]]. I. F. Ginzburg, K. A. Kanishev, M. Krawczyk and D. Sokolowska, Phys. Rev. D 82, 123533 (2010) arXiv:1009.4593 [hep-ph]].

[14] F. Mahmoudi, talk given at Prospects For Charged Higgs Discovery At Colliders (CHARGED 2012), 8-11 October, Uppsala, Sweden.

[15] N.G. Deshpande and E. Ma, Phys. Rev. D 18 (1978) 2574 .

[16] S. Kanemura, T. Kubota and E. Takasugi, Phys. Lett. B
313 (1993) 155 arXiv:hep-ph/9303263.

[17] A. G. Akeroyd, A. Arhrib and E. -M. Naimi, Phys. Lett. B 490, 119 (2000) hep-ph/0006035.

[18] M. Baak, M. Goebel, J. Haller, A. Hoecker, D. Ludwig, K. Moenig, M. Schott and J. Stelzer, Eur. Phys. J. C 72, 2003 (2012) arXiv:1107.0975 [hep-ph]].

[19] M. Baak, M. Goebel, J. Haller, A. Hoecker, D. Kennedy, R. Kogler, K. Moenig, M. Schott and J. Stelzer, arXiv:1209.2716 [hep-ph].

[20] See Fig. 12 in ATLAS' article in Ref. 3].

[21] We use the averages put forth in A. Arbey, M. Battaglia, A. Djouadi, F. Mahmoudi, arXiv:1211.4004, updated from ibid., JHEP 1209 107, (2012) arXiv:1207.1348 [hep-ph]].

[22] S. R. Coleman, Phys. Rev. D 15, 2929 (1977) [Erratumibid. D 16, 1248 (1977)]. S. Coleman, "Aspects of Symmetry," Cambrigde University Press, USA (1985).

[23] V. A. Rubakov, "Classical theory of gauge fields," Princeton, USA: Univ. Pr. (2002).

[24] I. Y. .Kobzarev, L. B. Okun and M. B. Voloshin, Sov. J. Nucl. Phys. 20, 644 (1975) [Yad. Fiz. 20, 1229 (1974)].

[25] M. Carena, S. Gori, I. Low, N. R. Shah and C. E. M. Wagner, arXiv:1211.6136 [hep-ph]. 